Presenting Author: Shueh Lim

Shueh Lim ${ }^{1}$, John Crowther ${ }^{2}$, Georgios Kontorinis ${ }^{2}$

${ }^{1}$ Royal Hospital Sick Children Glasgow,

${ }^{2}$ Queen Elizabeth University Hospital, Glasgow

Learning Objectives: Middle ear adenomas are rare benign tumours. Surgery remains the mainstay of treatment. Longterm follow up should be carried out. Middle ear adenoma should be included in the differential diagnosis of middle ear mass in patients with persisting, non-specific symptoms.

Introduction: Middle ear adenoma is a low-grade neoplasm with the potential for recurrence and metastases. Clinical and radiological findings can be misleading and often fail to provide the right diagnosis. Our objective is to present our experience over a 10 year period.

Methods: A retrospective review of all middle ear pathology at our tertiary referral centre with a catchment area of over three million over the last 10 years was carried out.

Results: In total, only three patients were identified (prevalence $1: 1,000,000)$, two male one female. Common symptoms of presentation included conductive hearing loss, aural pressure and autophony. Detailed imaging (computed tomography, magnetic resonance imaging complimented by angiography) was employed to better characterize the lesion and help with surgical planning. All patients underwent middle ear/ lateral skull base surgery. One patient had revision surgery for recurrence. Because of the neuroendocrine nature of ME adenomas, all patients remain under postoperative surveillance.

Conclusion: Our experience is similar to published literature. Adenomas of middle ear are indistinguishable benign tumours; surgical excision should be carried out to ensure complete excision.

doi:10.1017/S0022215116006101

\section{ID: IP114}

\section{Intracranial Complications from Chronic Otitis Media}

Presenting Author: Richard Locke

Richard Locke ${ }^{1}$, John Crowther ${ }^{2}$, William Taylor ${ }^{3}$, Georgios Kontorinis ${ }^{2}$

${ }^{1}$ Queen Elizabeth University Hospital, Glasgow, ${ }^{2}$ ENT Department, Queen Elizabeth University Hospital, Glasgow, ${ }^{3}$ Neurosurgery Department, Institute of Neurosciences, Queen Elizabeth University Hospital, Glasgow

Learning Objectives: Intracranial complications from middle ear disease Patterns of presentation Management of intracranial complications

Middle ear disease remains relatively common in the UK population with a previous study revealing the prevalence of inactive chronic otitis media to be $2.6 \%$ and active chronic otitis media to be $1.5 \%$ in the adult population. The incidence of intracranial complications from middle ear disease has fallen, however these life threatening complications are often diagnosed late and need to be treated aggressively.

We performed a retrospective analysis of patients referred to a tertiary neurosurgical centre with intracranial complications from both mucosal and squamous middle ear disease. The case notes and imaging were reviewed for comparison. Patient age, symptom duration, type of middle disease, management, complications and outcomes were determined. The results were then compared with a similar analysis performed at the same institution 20 years previously.

The majority of patients had active squamous otitis media. Previous surgical treatment of cholesteatoma did not prevent development of intracranial complications when there was recurrent disease. Meningitis was the predominant intracranial complication with venous sinus thrombosis and abscess rates lower. One patient developed a false aneurysm of the internal carotid artery from active mucosal otitis media.

There has been little change in the range of complications encountered over the past 20 years. For conditions such as false aneurysms, advances in interventional radiology have led to improved outcomes.

We present these cases to highlight the issues regarding diagnosis and management of patients with intracranial complications from chronic middle ear disease. High index of suspicion and aggressive surgical treatment remain essential as delay in diagnosis or treatment can be catastrophic.

doi:10.1017/S0022215116006113

\section{ID: IP115}

Cholesteatoma: How it grows and where it goes, and how we should therefore approach its surgery: An analysis of data collected prospectively on 516 cases.

Presenting Author: James Loock

James Loock

University of Stellenbosch

\section{Learning Objectives:}

Introduction: While all otologists operate on cholesteatoma, little analysis exists of the patterns of disease spread which logically should dictate surgical approaches. Nor has comparison been made between different parts of the world

Method: A detailed data-base was prospectively collected on every cholesteatoma surgery in Tygerberg Hospital between 2003 and $2016(\mathrm{n}=516)$. This included inter alia the cholesteatoma's origin from the tympanic membrane, and its presence/not in all parts of the mesotympanum, epitympanum and mastoid. This data is analysed for the various sites and subsites and the site of origin of the cholesteatoma.

Results: Surprisingly, the tympanic membrane origin was: pars flaccida $27 \%$, pars tensa posterior-superior marginal $29 \%$; pars tensa central $30 \%$; and indeterminate $13 \%$ (some had multiple origins). 
Cholesteatoma involved the epitympanum in $78 \%$, mesotympanum in $77 \%$, and mastoid in only $52 \%$. Subsite involvement in the epitympanum was: posterior $86 \%$; middle, superficial to ossicles $69 \%$; middle deep to ossicles $54 \%$; anterior $65 \%$, and anterior epitympanic recess $9 \%$.

In the mesotympanum it was central over promontory in $76 \%$; posterior-superior in $72 \%$; in the facial recess and sinus tympani in $48 \%$, anterior in $22 \%$ and in the hypotympanum in $12 \%$.

In the mastoid it was restricted to the antrum in $52 \%$ and more widely in $26 \%$.

Differences are described in the pattern of spread dependent on the site of origin from tympanic membrane.

Conclusion: This data shows cholesteatoma is primarily a condition of the meso- and epitympanum. One thus needs to be able to remove it from the complex subsites of the middle ear, including retrotympanum, and rehabilitate the mesotympanum. The frequent involvement of the ossicles in the epitympanum demands techniques to mitigate recidivism here. Our South African data is significantly different from what the literature describes - the question is whether this disease is different in different regions.

\section{doi:10.1017/S0022215116006125}

\section{ID: IP116}

\section{Meeting Medical Expectations in Pediatric Cholesteatoma Surgery - Revisited}

\section{Presenting Author: Michal Luntz}

\author{
Michal Luntz, Riad Khnifies, Noam Yehudai \\ Bnai Zion Medical Center, Technion - The \\ Bruce Rappaport Faculty of Medicine, Haifa, \\ Israel
}

Learning Objectives: to establish realistic expectations in pediatric cholesteatoma in the era of non-EPI-diffusion weighted MRI.

Introduction: Cholesteatoma is a struggle for a safe and convenient condition with the least possible surgeries in the presence of a disease that tends to re-create after complete removal due to the compromised ME physiology, as well as the possibility of residual disease.

Methods: Data collected from consecutive pediatric cholesteatoma surgeries performed by a single surgeon (ML) between 2001 and 2005 and between 2011 and 2015. Type and number of surgeries performed in each group over the follow up period before (2001 and 2005) and after (2011 and 2015) the introduction of non-EPI-diffusion weighted MRI were compared. Revision surgery was also performed in both groups at any point when recurrent cholesteatoma is detected by routine follow-up otoscopy (4-6 weeks, $3 \mathrm{~m}$, and every $6 \mathrm{~m}$ thereafter).

Results: There were no significant medical or surgical complications in either group. $54.5 \%$ of the children operated between 2001-2005 ended with a CWD mastoidectomy condition (radical cavity) after a mean follow up of $5.8 \pm 3.8$. None of the children operated during
2011-2015, after the introduction of routine periodic post -operative non-EPI diffusion MRI follow-up ended at this point or expected to have at any point a radical mastoidectomy.

Conclusions: Currently, radical mastoidectomy with meatoplasty should not be used as one of the routine surgical options in pediatric cholesteatoma. Follow up (clinical and imaging) after cholesteatoma is absolutely mandatory, without it, children with cholesteatoma are exposed to a very significant and unnecessary risk. In cases of repeated, de-novo re-creation of cholesteatoma, or repeated infection in an existing radical cavity, a CWD mastoidectomy with blind sac obliteration of the EEC and the ME cleft is also a very practical option in certain cases, as it eliminates denovo re-creation of cholesteatoma.

doi:10.1017/S0022215116006137

\section{IP117}

\section{Hearing in Patients with Cholesteatoma:} Facing Reality

Presenting Author: Michal Luntz

Michal Luntz, Riad Khnifies, Noam Yehudai

Bnai Zion Medical Center, Technion - The

Bruce Rappaport Faculty of Medicine, Haifa, Israel

Learning Objectives: to confront the otologic community with the non-optimal hearing of individuals with controlled cholesteatoma.

Introduction: Goals of management in cholesteatoma are to create a safe ear (avoiding complications), to achieve a dry and convenient ear and to reach the best possible hearing restoration. Although hearing restoration is not defined as the first priority for treatment in cholesteatoma, it may have serious long-term effects on patient's life.

Methods: The study included two study groups. In one group there were 260 consecutive cholesteatoma surgeries, 128 adults and 132 children. The mean group follow-up was $6.6 \pm 12.8$ yrs. Hearing thresholds were analyzed according to type of surgery performed and age of the patients (children Vs adults). The other group includes 39 ears of children who were operated after the introduction of routine use of none EPI-diffusion weighted MRI and the complete avoidance of traditional radical cavities. In this group follow up was much shorter (Mean $1.4 \pm 1.3$ yrs.)

Results: Mean group air conduction (AC) PTA after canal wall up procedures were $38 \mathrm{~dB}$ in children and $55 \mathrm{~dB}$ in adults. Mean group AC-PTA after canal wall down procedures were $70 \mathrm{~dB}$ in children and $60 \mathrm{~dB}$ in adults. Group AC-PTA after canal wall up procedures were $38 \mathrm{~dB}$ in children and $55 \mathrm{~dB}$ in adults. In the group with radical mastoidectomy and reconstruction of the EEC and mastoid obliteration mean group AC-PTA was $40 \mathrm{~dB}$ as compared to $60 \mathrm{~dB}$ in the canal wall down cases which were left as radical cavities. 